\section{Assessment of iron deficiency in rheumatoid arthritis}

In a recent survey of 100 consecutive patients with rheumatoid arthritis (RA) attending a rheumatology outpatient department, over $75 \%$ had received a course of oral iron during their disease and $40 \%$ multiple (up to 12) courses (D R Blake and D G I Scott, unpublished observations). Since these figures almost certainly do not indicate the true incidence of iron deficiency, we have assessed the value of standard haematological indices of iron state to predict low iron stores. Serum ferritin concentration is a particularly sensitive index of low iron stores in $\mathrm{RA}^{1}$ and has, therefore, been accepted as the absolute iron state in our study. Patients with a low serum ferritin concentration were further investigated to assess the cause of their iron deficiency.

\section{Patients, methods, and results}

The standard laboratory profile used to predict iron state in clinical practice includes haemoglobin concentration; packed cell volume; the red-cell variables - mean cell volume, mean cell haemoglobin, and mean cell haemoglobin concentration (measured with a Coulter $S$ automatic cell counter); and serum iron concentration and iron binding capacity (determined with a Technicon autoanalyser by the triazine method).

These variables were measured in 110 patients ( $34 \mathrm{men}, 76$ women), who fulfilled the criteria of the American Rheumatism Association for definite or probable $\mathrm{RA}^{2}$ and who were currently attending medical and rheumatological outpatient departments. A sample of serum was stored at $-20^{\circ} \mathrm{C}$ for independent estimation of ferritin concentration (immunoradiometric assayGammadab ${ }^{125}$ I, Travenol Lab).

Of the 110 patients studied, seven ( $6 \%$, three men and four women) had low iron stores as judged by serum ferritin concentration $(<12 \mu \mathrm{g} / \mathrm{l})$. The table shows the standard haematological variables (mean $\pm 1 \mathrm{SD}$ ) for these patients in contrast with the remainder, whose ferritin concentrations were normal or raised. Of the eight patients with low ferritin concentrations, two had a haemoglobin concentration above the accepted lower limit of normal for their age and sex. ${ }^{3}$ Fourteen patients had recently been prescribed iron, and subsequent estimations of their serum ferritin concentration showed that seven had values above, six had values within, and only one had a value below the normal range. Further analysis of the results in the patients with a low serum ferritin concentration detected no combination of variables that could have reliably or selectively predicted a low serum ferritin concentration.

Investigation of the eight patients with a low serum ferritin concentration disclosed that two had acute duodenal ulcers, one a benign gastric ulcer, and one presumed bleeding from a hiatus hernia. In two patients no specific cause for the low serum ferritin concentration was found, though both were women with a history of menorrhagia.

\section{Comment}

A moderate anaemia is the commonest systemic manifestation of $\mathrm{RA}$, and its severity reflects the activity of the disease. Though the anaemia is often hypochromic and sometimes microcytic, it reflects the inability of the reticuloendothelial cells to release sequestered iron and, less commonly, a genuine iron deficiency. The avid retention of iron by the reticuloendothelial system is reflected by a rise in serum ferritin concentration, which has been observed to be high in inflammatory states, ${ }^{4}$ and the association between serum ferritin concentration and the activity of rheumatoid disease needs to be explored further.

The concentration of serum ferritin below which iron deficiency is said to occur is normally $12 \mu \mathrm{g} / \mathrm{l}^{1}$ Almost certainly this value is inappropriate for those with RA and should be adjusted upwards. We have shown, however, that even if a concentration of under $12 \mu \mathrm{g} / 1$ is accepted, at least $6 \%$ of our patients were genuinely iron deficient, and that none of the routine variables of iron state adequately predicted this group. The incidence of other diseases in patients with a low serum ferritin concentration is disquieting, and the need for further investigation is clear. All patients in this study were receiving nonsteroidal anti-inflammatory drugs capable of inducing gastrointestinal bleeding, but these should not be readily accepted as the cause of a low serum ferritin concentration.

The indiscriminate use of oral iron in patients with RA is inappropriate and dangerous. An estimation of serum ferritin concentration could usefully be incorporated as a routine measurement in the assessment of the rheumatoid patient, the cost being offset by a more rational prescribing policy.

We are grateful to Dr M Thompson (Newcastle upon Tyne) and Dr P A Bacon (Bath) for their support and advice.

${ }^{1}$ Bentley DP, Williams P. Serum ferritin concentration as an index of storage iron in rheumatoid arthritis. $\mathcal{F}$ Clin Pathol 1974;27:786-8.

2 Ropes MW, Bennett GA, Cobb S, Jacox R, Jessar R. Revision of diagnostic criteria for rheumatoid arthritis. Bull Rheum Dis 1958;9:175-6.

${ }^{3}$ Diem K, Lentner C, eds. Documenta Geigy-Scientific tables. 1970:617.

${ }^{4}$ Lipschitz DA, Cook JD, Finch CA. A clinical evaluation of serum ferritin as an index of iron stores. $N$ Engl $\mathcal{F}$ Med 1974 ;290:1213-6.

(Accepted 5 December 1979)

Royal National Hospital for Rheumatic Diseases, Bath BA1 1RL

D R BLAKE, MB, MRCP, senior registrar in rheumatology (formerly at Royal Victoria Infirmary, Newcastle upon Tyne)

D G I SCOTT, MB, MRCP, registrar in rheumatology

Department of Child Health and Medicine, Royal Victoria Infirmary, Newcastle upon Tyne NE1 4LP

E J EASTHAM, MB, MRCP, senior registrar in paediatrics

H RASHID, MB, FCPS (BANGLADESH), honorary research associate

\section{Raised blood urea concentration indicates considerable blood loss in acute upper gastrointestinal haemorrhage}

Estimates of the amount of blood lost during acute upper gastrointestinal haemorrhage are known to be inaccurate because of the indirect and inadequate indicators available. ${ }^{12}$ The blood urea concentration has long been known to rise after upper gastrointestinal bleeding, but its value as an indicator of the volume of blood lost has been questioned because of uncertainty about the pathophysiology of its rise.

We undertook this study to see whether the blood urea concentration on admission allows prediction of the volume of acute blood loss. We also compared the creatinine clearance on admission with that at the time of discharge to establish whether hypovolaemia or absorption of blood are predominantly responsible for the rise. ${ }^{34}$

\section{Patients, methods, and results}

We studied 57 patients admitted to hospital with a history suggesting upper gastrointestinal bleeding. Estimations of haemoglobin concentration, mean corpuscular volume, mean corpuscular haemoglobin, and blood urea and creatinine concentrations were made on a sample of blood taken on admission, and on samples taken 24,48 , and 72 hours after admission and on the day before discharge. Thirty-eight patients had creatinine clearance

Haematological variables in 110 patients with rheumatoid arthritis having low or normal iron stores (as shown by serum ferritin concentration). Results are given as means $\pm 1 S D$ with ranges in parentheses

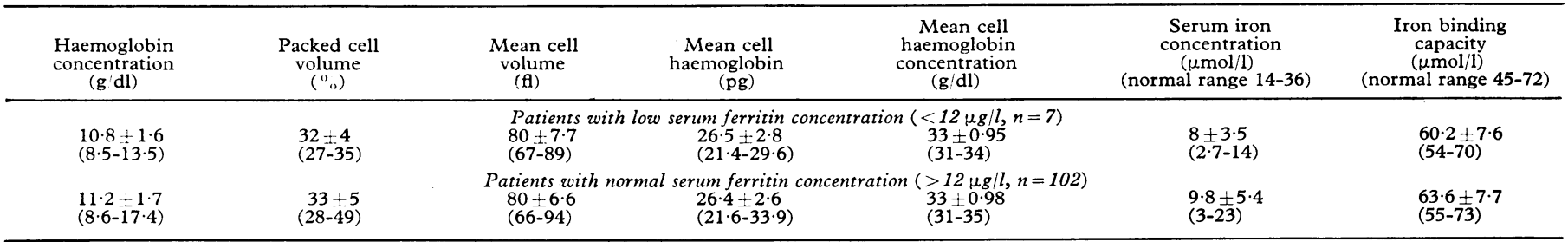


measurements made on admission and immediately before discharge. The volume of blood lost was calculated by adding the volume transfused (in pints) to the difference between the haemoglobin concentration before discharge and an accepted normal haemoglobin concentration (14 $\mathrm{g} / \mathrm{dl}$ for men, $13 \mathrm{~g} / \mathrm{dl}$ for women). Statistical analysis was carried out using Spearman's rank correlation.

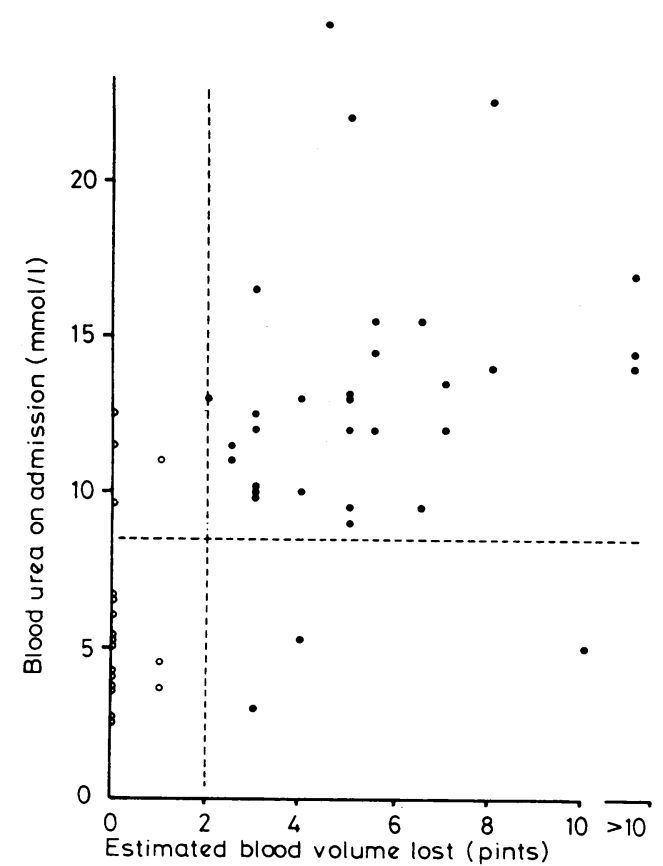

Blood urea concentration on admission in 51 patients with suspected acute upper gastrointestinal haemorrhage. Eighteen $(\mathrm{O})$ had only minor blood loss, whereas $33(0)$ had considerable blood
loss. Traditional units to SI: 1 pint $=0.571$.

Of the 57 patients, four were excluded because of chronic haemorrhage indicated by reduced mean corpuscular volume, mean cell volume, and iron stores; two were excluded because of abnormal findings in liver function tests. No patient had a persistently raised creatinine concentration suggesting chronic renal disease. Of the remaining 51 patients, 33 had evidence of considerable bleeding (more than two pints $(1 \cdot 14 \mathrm{l})$ of blood), whereas 18 had no evidence of severe haemorrhage. There is a highly significant correlation between urea concentration on admission and estimated volume of blood lost $(R=0.72, p \leqslant 0.001$ ) (figure).

Of 33 patients with considerable haemorrhage, 30 had a urea concentration temporarily above $8.5 \mathrm{mmol} / \mathrm{l}$, whereas only three had a urea concentration of less than $8.5 \mathrm{mmol} / \mathrm{l}$. Of the 18 patients without considerable bleeding, 14 had no rise in urea concentration, whereas four had a temporary rise. The urea concentration on admission did not relate to any difference between creatinine clearance immediately after the bleed and on discharge, and there was no correlation between the difference in creatinine clearance on these two occasions and pints of blood lost.

\section{Comment}

These results suggest that a blood urea concentration on admission that is over $8.5 \mathrm{mmol} / 1$ indicates considerable blood loss and that the blood urea concentration correlates with the volume of blood lost.

Two of the three patients with haemorrhage and no rise in urea concentration justify discussion. One presented with acute haematemesis requiring transfusion of three units of blood but at no stage were his stool samples positive for occult blood, and presumably he never had blood in his upper gastrointestinal tract to be absorbed. The other bled from a Crohn's ulcer in the jejunum just proximal to a jejunocolic anastamosis. We assume that his haemorrhage, which required transfusion of 12 units of blood, occurred at a site too distal for blood products to be absorbed. These two patients support the hypothesis that the main cause of uraemia after gastrointestinal bleeding is absorption of blood products. Our finding of no significant difference between creatinine clearance immediately after the bleed and on discharge is in keeping with this. Four patients had a raised urea concentration and no appreciable bleeding. Three of these had a history of recurrent vomiting, and their urea concentration fell to normal within 24 hours on rehydration, in contrast to the 48 hours or more for patients with considerable bleeding, suggesting that dehydration alone accounted for the uraemia of the three without appreciable bleeding. The fourth patient had melaena from an active duodenal ulcer and has since been shown to be polycythaemic, so that our method of calculation underestimated his blood loss.

${ }^{1}$ Burnett W. Diagnosis and treatment of haematemesis. Med $\mathcal{F}$ Aust 1966; 1:1102-4.

2 Mailer C, Goldberg A, Haiden R McG, Grey-Thomas I, Burnett W. Diagnosis of upper gastrointestinal bleeding. Br Med f 1965;ii:784-9. ${ }_{3}^{3}$ Meyler L. Post-haemorrhagic uraemia. Acta Med Scand 1935;87:313-25. 4 Christiansen T. Uraemia as cause of death in massive haemorrhage from peptic ulceration. Acta Med Scand 1935;85:333-45.

(Accepted 3 December 1979)

\section{Whittington Hospital, London N19 5NF}

C W PUMPYREY, MRCP, medical registrar (present address: St Pancras Hospital, London NW1 OPE

E R BECK, FRCP, consultant physician
ONE HUNDRED YEARS AGO There is in the present case, perhaps, nothing about the mode of origin, sequence of growth, or period of recurrence, generally, that is not sufficiently known; but, in reference to the particular structures concerned, a similar chain of events appears not to have been often recorded.

Mrs C H of Bury, then fifty-three years of age, and the wife, now widow, of a teacher long invalided through paralysis and in straitened circumstances, came under treatment in May 1874. A spark of coal from the fire-grate had struck her right eye about a year before. A papillose luxuriance of moderate extent in the scleral conjunctiva, at the temporal side of the cornea, was the seat of pain, from which she came to seek relief. The growth was removed; and a small particle of coal was found at the bottom of it, rather firmly fixed in the sclerotic. It was taken out, and the patient went home the same day. Eight months afterwards-on February 11 th, 1875-the size and painfulness of the recurrent growth called for fresh excision. This time, a stay of three weeks became necessary before the patient was able to return home. The interval between the next and third removal of the considerably enlarged but still practically episcleral neoplasm was only half of the previous one; it took place on July 19th, 1875 . The patient required to be attended for four weeks before she could be discharged; and it was now foreseen that, in case of return, the eye would have to be removed. This came to pass six months afterwards, in January 1876. The eye, as an organ of sight, was as perfect as its fellow; simply presbyopic, in accordance with the patient's age of life. There was no protrusion; no interference with the movements of the eye; hence no diplopia. But there was much pain, and no longer a chance of detaching the growth from the shell of the eye without fatal injury to the latter. Owing to the patient's cardiac affection, which was of long standing, the attempt that was made to induce anaesthesia had to be desisted from. Dr Dreschfeld, who was going to be at the trouble of examining the eye, was present at the operation. The opportunity for obtaining from the living body an eyeball in itself, or at least optically, so thoroughly sound, being exceedingly rare, it is matter for regret that the discovery of the visual purple was not made or published until some time later on. Dr Dreschfeld found the growth to be a roundcelled sarcoma entirely confined within the sclera. Five weeks after the operation, the patient returned home. For upwards of two years thereafter, whilst I saw her, on an average, every six months, no trace of recurrence could be noticed. It is true that a painful sensation was said to be occasionally felt; which was, however, referred to the front part of the orbital roof. It was not before November 1878 that the mere vestige of a nodular swelling within the margin of the lower lid near the inner angle, being also the seat of pain, began to arouse my fears. Nine months afterwards, a swelling of the size of a small walnut, slightly tuberculated, had come to occupy the lower lid beneath its margin, the latter being now entirely free. It has since grown rather rapidly. Some patches of xanthelasma upon the surface of the swelling now appear, of a pearly white, from the expansion of the integument. The latter, for some little time past, has threatened to give way at a spot more highly reddened than the rest. Removal of the new and painful tumour appears urgent. The right eye remains perfectly sound.

The case, as here related, was presented at the last November meeting of the Manchester Medical Society. In the reappearance of the disease, originally developed in the sclerotic, after a lapse of two years, in structures so dissimilar as the palpebral, there is, perhaps, nothing extraordinary but the proximity of the latter. (British Medical fournal, 1880.) 